\title{
ERRATUM
}

Lan Kluwe · Stefan M. Pulst · Johannes Köppen

Victor-F. Mautner

\section{A 163-bp deletion at the C-terminus of the schwannomin gene associated with variable phenotypes of neurofibromatosis type 2}

Hum Genet (1995) 95:443 -446

The title of the above article has been changed as follows:

\section{A 163-bp deletion in the neurofibromatosis 2 (NF2) gene associated with variant phenotypes}

L. Kluwe $\cdot$ J. Köppen

Laboratory for Brain Tumor Biology,

Department of Neurological Surgery,

University Hospital Eppendorf, Martinistrasse 52,

D-20246 Hamburg, Germany

S. M. Pulst

Neurogenetics Laboratory and Division of Neurology,

Cedars-Sinai Medical Center,

University of California at Los Angeles,

8700 Beverly Boulevard, Los Angeles, CA 90048, USA

V.-F. Mautner (凶)

Neurologische Abteilung, Allgemeines Krankenhaus Ochsenzoll,

Langenhorner Chaussee 560, D-22419 Hamburg, Germany 